Rev SINAPSIS, Vol. 6, Nº 1, Junio 2015

ISSN $1390-7832$

\title{
El Idioma Inglés como segunda lengua en los profesionales de la carrera de enfermería El inglés para enfermeros
}

Marianela San Lucas Marcillo. Mg.EII (1)

Marieta Azua Menéndez. Mg. EI ( 2 )

José Tigua Anzules. Mg. EII ${ }^{(3)}$

(1) Universidad Estatal del Sur de Manabí, Jipijapa, Ecuador

(2)Universidad Estatal del Sur de Manabí, Jipijapa, Ecuador

(3) Universidad Estatal del Sur de Manabí, Jipijapa, Ecuador

Contacto: marian_san2008@hotmail.com

Receptado: 05/03/2015 Aceptado: 02/05/2015

\section{Resumen:}

La presente investigación se realizó en la Universidad Estatal del Sur de Manabí, básicamente en los profesionales y estudiantes de la carrera de enfermería. El nuevo perfil competencial exige que posean competencias específicas en inglés hablado y escrito. El aprendizaje del idioma Inglés tiene como propósito específico adquirir características especiales que vayan con la carrera, en comparación con el inglés general, ya que no es suficiente. Por otra parte, ellos consideran importante el aprendizaje del idioma inglés, indicando también la inconformidad en aprender pero que de acuerdo a las necesidades actuales se debe tener en cuenta cómo ha influenciado el aprendizaje de este idioma, por lo tanto, es necesario adquirir conocimiento de este lenguaje con fines específico, para que los estudiantes y profesionales de la carrera de enfermería no presenten barreras que obstaculicen su vida laboral. A pesar de que todos coinciden en la importancia del dominio del idioma Inglés en el mundo actual, uno de los objetivos del profesional es ser motivado a comprender y aprender el inglés con fines específicos, cuyo campo es el idioma inglés con enfoque en el área de la salud. De acuerdo a los métodos utilizados en esta investigación fueron: el histórico-lógico, inductivo-deductivo y la observación, encuestas a estudiantes y entrevistas a los profesionales en enfermería. Se obtuvo como resultado que el idioma ingles es fundamental como segunda lengua hablada y escrita.

Palabras clave: Propósito específico, competitivo, segunda lengua, idioma inglés, imprescindible, obstáculo 
ISSN $1390-7832$

Rev SINAPSIS, Vol. 6, № 1, Junio 2015

\section{Abstract}

\section{English as second language in the nursing professional}

This research was performed at the Estatal del Sur de Manabí University, basically professionals and students of the nursing career. The new competence profile required with specific competence in spoken and written English. Learning the English language has a specific purpose acquire special features that go with the race, compared to general English, as it is not enough. Moreover, they consider important to the English language, also indicating the disagreement to learn but that according to current needs should take into account how learning has influenced the language, therefore, it is necessary to acquire knowledge This language with specific purposes for students and career professionals nursing do not present barriers to their working life. While all agree on the importance of English language proficiency in today's world, one of the objectives of the professional is to be motivated to understand and learn English for specific purposes, whose field is English with a focus on the area the health. According to the methods used in this research it was: the historical and logical, deductive and inductive observation, student surveys and interviews with nursing professionals. It resulted that the English language is essential as a second spoken and written language.

Keywords: Specific purpose, competitive, second language, English language, indispensable obstacle

\section{Introducción}

El idioma inglés es considerado actualmente el más importante a nivel mundial. Se puede decir que es el principal elemento de comunicación entre culturas muy diversas, mucho más para los profesionales de enfermería, porque no solo es importante poseer conocimientos en inglés, sino competencias comunicativas para aplicarlas en sus actividades profesionales, en el manejo de informaciones complejas y en otras actividades que requieren de la comunicación. (Quezada, Finalet, Recino, \& Yagima, 2015)

Por otra parte, los profesionales de enfermería tienen limitaciones en el uso de la lengua inglesa, no desarrollando así las competencias comunicativas que requiere la sociedad del siglo XXI, (Camacho B. R., Barquero, Isabel, \& Merino, 2013) 
Rev SINAPSIS, Vol. 6, No 1, Junio 2015

ISSN $1390-7832$

En la actualidad el inglés se refleja en investigación, el manejo de manuales de instrucciones de aparatos e instrumentos que se encuentran en los hospitales, ambulatorios, u otros Centros de Salud, el manejo de ordenadores y programas informáticos y la interacción con el paciente extranjero y/o familiares o amigos de éste.

Según (Niño, 2013) considera que para sobrevivir en este mundo competitivo, todos debemos ser capaces de asumir nuevos retos y oportunidades. A través del uso de internet y los teléfonos celulares, el mundo se ha convertido en una pequeña aldea, donde el primero que recibe la información sobresaldrá ante aquellos que nunca la reciban o la reciban muy tarde. ¿Qué idioma nos permite una comunicación a gran escala? ... El inglés, por supuesto.

Cuando se trata del manejo de manuales de instrucciones de aparatos e instrumentos se debe tener en cuenta que, dado el rápido avance de la tecnología en todos los campos incluidos el de la medicina, llegan constantemente a los hospitales, clínicas, ambulatorios y otros Centros de Salud, suelen venir la mayoría en inglés.

Al respecto (Hernandez, Cardenas, Sanchez, \& Torres, 2012) opinan que a pesar de la diversidad de métodos utilizados y su flexibilidad en cuanto a su aplicación, se constata que existen dificultades en el desarrollo de las habilidades comunicativas del personal de enfermería, concurriendo a las necesidades de aprendizaje e intereses en estos profesionales, asegurando la capacidad de desempeñarse con éxito en las diferentes esferas de su vida laboral.

Este proceso se manifiesta dentro del contexto latinoamericano, donde el potencial crecimiento de la disciplina de enfermería requiere una adaptación del perfil profesional a las nuevas necesidades y demandas de la sociedad, acercando la realidad académica y profesional (Camacho B. R., Barquero, Mariscal, \& Merino, 2013)

Indicando la diferencia existente en la formación en enfermería en América Latina, ha sido recogida por diversos autores en el cual también reflejan la necesidad de unificar criterios, especialmente los relacionados con la investigación y el uso de la evidencia científica. 
Rev SINAPSIS, Vol. 6, Nº 1, Junio 2015

Desde la perspectiva pedagógica, según (Montané, Trujillo, Cruz, Carlos, \& Bosque, 2012), se ha tenido en consideración una pedagogía socialista, que garantiza el desarrollo integral de la personalidad del estudiante y dentro de ella se ha tenido en cuenta: La teoría de la competencia comunicativa, de los actos del habla, del contexto y el proceso de enseñanza-aprendizaje del inglés con fines específicos.

En este caso (Camacho B. R., Barquero, Mariscal, \& Merino, 2013) indican que es imprescindible plantear nuevas estrategias del inglés para potenciar la calidad de aprendizaje y organizarlos de manera que permitan un máximo aprovechamiento de capacidades cognoscitivas de los estudiantes y desarrollen sus posibilidades para trabajar de forma independiente, preparándolos para adquirir los conocimientos por sí mismos, así como aplicarlos de manera efectiva, original y creadora en el mundo laboral.

De acuerdo a las opiniones de los diferentes autores citados, la opinión de (Niño, 2013) es la más acertada ya que considera que para sobrevivir en este mundo competitivo, todos deben ser capaces de asumir nuevos retos y oportunidades alcanzando así una comunicación en Ingles a gran escala y del cual se debe se tiene que aprender..

Con la competencia comunicativa se modifica el papel tanto del profesor como del alumno. El profesor es un facilitador del aprendizaje de los estudiantes que promueve la creación de situaciones y tareas comunicativas. El alumno es un profesional en potencia que práctica el idioma relacionado con el perfil profesional con que se forma. (Pibaque, Quevedo, \& Colunga, 2015)

A partir de la experiencia en el aula en la carrera de enfermería de la Universidad Estatal del Sur de Manabí, el contraste entre lo que se enseña y lo que se practica suele provocar en los alumnos una gran confusión, uno de los aspectos señalados por los participantes de la mencionada carrera es que para cumplir las exigencias y al mismo tiempo garantizar una buena preparación técnica y profesional, se hace imprescindible abandonar las costumbres y tradiciones en lo referente a las formas organizativas de la docencia, los roles del estudiante y del profesor el cual resulta evidente el viejo paradigma de enseñanza tradicional. 
Rev SINAPSIS, Vol. 6, No 1, Junio 2015

ISSN $1390-7832$

A pesar de que todos coinciden en la importancia del dominio del idioma Inglés en el mundo actual, uno de los objetivos del profesional es ser motivado a comprender y aprender el inglés con fines específicos, donde el campo es el idioma inglés con enfoque en salud.

El aporte teórico radica en una concepción teórica donde se develan las relaciones que fundamentan la estrategia de enseñanza-aprendizaje del idioma inglés en profesionales de enfermería y el aporte práctico se precisa en la metodología, sustentada en una concepción enfocada al desarrollo de prácticas como dramatizaciones, que es factible de llevarse a la práctica con los estudiantes que están inmersas en las actividades de salud, para potenciar la comunicación con los pacientes de habla inglesa. El impacto social de la investigación radica en que se favorecen todos los profesionales que se dedican a esta rama y a la vez para que puedan desempeñar mejor en su labor y comunicación con los pacientes extranjeros.

\section{Materiales y métodos}

La competencia comunicativa no se limita al dominio del inglés, sino que implica también la capacidad que le permite al estudiante saber cómo y cuándo utilizar los conocimientos, porque el dominio de la lengua no se reduce solo a un saber, sino que también consiste en un saber hacer, en un saber ejecutar para un mayor comunicación.

La investigación se realizó con profesionales y estudiantes de la carrera de enfermería de la Universidad estatal del Sur de Manabí.

La población estuvo constituida por 85 estudiantes del cuarto semestre de la carrera de enfermería y del cual fueron encuestados 70 estudiantes y 5 profesionales en la especialidad de la carrera de enfermería. Estas encuestas y entrevistas permitieron conocer las opiniones acerca el nivel de las habilidades comunicativas alcanzado por los estudiantes y profesionales en idioma inglés con propósitos específicos y también la muestra (70) quedó determinada entre los estudiantes que demostraron un poco interés a las habilidades comunicativas en idioma inglés con propósito específico, indicando que ya la estudian porque forma parte de la malla curricular y el cual tienen que aprobarla. 
Rev SINAPSIS, Vol. 6, Nº 1, Junio 2015

Por otra parte consideran importante el aprendizaje en la enseñanza del idioma inglés con propósitos específicos, en la actualidad para los egresados de la carrera de Enfermería, contenidos posibles a tener en cuenta y cómo han influido otros factores tales como la amplia diversidad educativa en cuanto a los niveles de formación en el aprendizaje de este idioma, las dimensiones a tener en cuenta, y las barreras que obstaculizan el desarrollo de las habilidades comunicativas en los profesionales en Enfermería.

Para la realización del estudio, se partió de una sistematización teórica de los trabajos más importantes en la temática, se buscó en una amplia bibliografía, tomándose en consideración los antecedentes relacionados con el idioma inglés como segunda lengua en los profesionales de enfermería donde requieren de la comunicación.

\section{Métodos teóricos:}

Análisis-síntesis e inducción-deducción que permitieron la interpretación de la información bibliográfica y de los datos estadísticos; además, posibilitaron la caracterización del objeto y el campo a partir del análisis de los fundamentos teóricos que los sustentan; El histórico-lógico viabilizó la caracterización de los modelos explicativo-descriptivos utilizados en la estrategia de la construcción textual escrita del idioma inglés y en la disciplina del objeto de estudio que anteceden así como de las tendencias actuales en su desarrollo; El método sistémico estructural - funcional se utilizó en la explicación del objeto de la investigación y en la modelación de la concepción teórica, mediante la determinación de los subsistemas que la conforman y de las relaciones que se revelan dentro y entre cada uno de ellos, así como en el establecimiento, estructuración y funcionalidad de la metodología.

De los métodos empíricos: se aplicaron observaciones en el aula y fuera de esta, encuestas y entrevistas, para evaluar el estado actual de la competencia comunicativa del inglés y su formación en estudiantes del área de enfermería.

\section{Resultados}

Durante el período de investigación y aplicando encuestas a los alumnos y entrevistas a los profesionales de la carrera de enfermería, fueron corroborados a partir de la triangulación metodológica realizada y se pudo constatar que de 85 estudiantes (población), 70 (muestra) fueron 
Rev SINAPSIS, Vol. 6, Nº 1, Junio 2015

encuestado en la carrera de enfermería de la Universidad Estatal del Sur de Manabí , donde se elaboraron figuras con datos relacionados con las variables que a continuación se detallan: En la figura No.1 hace referencia de que si ¿considera Ud. que el dominio del idioma ingles debe primar en los profesionales en enfermería? y, de acuerdo a su tabulación se determina que un 93\% manifestó que si, un $7 \%$ dijo que no y un $0 \%$ no sabe no contesta, por lo que se indica que ellos si presentan interés en aprender este idioma extranjero (Inglés) ya que piensan que en su actividad profesional sería necesario.

\section{Figura No.1}

Considera que el dominio del idioma ingles debe primar en los profesionales en enfermería

\begin{tabular}{lcc}
\hline \multicolumn{1}{c}{ ALTERNATIVA } & FRECUENCIA & PORCENTAJE \\
\hline $\mathrm{Si}$ & 65 & $93 \%$ \\
$\mathrm{No}$ & 5 & $7 \%$ \\
No sabe/No contesta & 0 & $0 \%$ \\
\multicolumn{1}{r}{ TOTAL } & 70 & $100 \%$ \\
\hline
\end{tabular}

Fuente: "Carrera de Enfermería de la Universidad Estatal del Sur de Manabí

Elaborado por: Mg. Marianela San Lucas Marcillo, Mg. Marieta Azúa Menéndez, Mg. José Tigua Anzules

Continuando con el análisis de la figura No. 2 referente a ¿si consideran fundamental escuchar, leer, hablar y escribir a la hora de aprender una nueva lengua?, muestra como resultado que un $100 \%$ manifiestan que si son necesarias las habilidades comunicativas, un $0 \%$ dice que no y de la misma manera No sabe/No contesta. Cabe recalcar que las habilidades comunicativas (listening, Reading, speaking and Writting) se consideran fundamental a la hora de aprender una nueva lengua, sin perder de vista los elementos gramaticales y situacionales, porque la competencia comunicativa determina la capacidad del hablante de la lengua para interactuar, intercambiar e interpretar significados con un modo adecuado en una situación real.

\section{Figura No.2}

Considera que el dominio del idioma ingles debe primar en los profesionales en enfermería

\begin{tabular}{lccc}
\hline & ALTERNATIVA & FRECUENCIA & PORCENTAJE \\
\hline $\mathrm{Si}$ & 70 & $100 \%$ \\
$\mathrm{No}$ & 0 & $0 \%$ \\
\hline
\end{tabular}


Rev SINAPSIS, Vol. 6, Nº 1, Junio 2015

\begin{tabular}{ccc}
\hline No sabe/No contesta & 0 & $0 \%$ \\
TOTAL & 70 & $100 \%$ \\
\hline
\end{tabular}

Fuente: "Carrera de Enfermería de la Universidad Estatal del Sur de Manabí

Elaborado por: Mg. Marianela San Lucas Marcillo, Mg. Marieta Azúa Menéndez, Mg. José Tigua Anzules

En la figura No. 3 se analiza los resultados de la pregunta \& A Ud. le gustaría aprender el idioma ingles mediante capacitaciones, cursos y talleres para uso laboral en lo profesional?, el 65\% manifiesta que si se encuentran motivados en aprender el idioma, un $7 \%$ no les gusta y un $0 \%$ no sabe no contesta, determinando que los estudiantes de la carrera de enfermería de la Universidad Estatal del Sur de Manabí deben reforzar las habilidades cognitivas y estar íntimamente relacionada con lo que el estudiante debe haber adquirido en el proceso educativo para enfrentarse a su vida profesional.

\section{Figura No.3}

Le gustaría aprender el idioma ingles mediante capacitaciones, cursos y talleres para uso laboral en lo profesional

\begin{tabular}{lccc}
\hline & ALTERNATIVA & FRECUENCIA & PORCENTAJE \\
\hline $\mathrm{Si}$ & 65 & $93 \%$ \\
$\mathrm{No}$ & 5 & $7 \%$ \\
No sabe/No contesta & TOTAL & 0 & $0 \%$ \\
\multicolumn{2}{r}{$\quad$} & 70 & $100 \%$ \\
\hline
\end{tabular}

Fuente: "Carrera de Enfermería de la Universidad Estatal del Sur de Manabí

Elaborado por: Mg. Marianela San Lucas Marcillo, Mg. Marieta Azúa Menéndez, Mg. José Tigua Anzules

En la entrevista realizada a los profesionales se pudo conocer que $90 \%$ manifestaron no contar con la suficiente preparación en inglés que les permita una adecuada conversación con un paciente extranjero.

Para la realización del referido estudio se asumieron los siguientes indicadores: Contenidos que se privilegian para la formación profesional, contribución de la enseñanza aprendizaje del inglés al desarrollo de competencias comunicativas y metodología de enseñanza aprendizaje.

\section{Discusión}


Rev SINAPSIS, Vol. 6, Nº 1, Junio 2015

ISSN $1390-7832$

Con respecto a que si el idioma Inglés está dentro de sus necesidades, la mayoría consideraron que su aprendizaje es importante para intercambiar conocimientos con otros colegas, pacientes, en eventos científicos y otros espacios de encuentros relacionados con la profesión.

En relación con el tipo de actividades que consideran necesarias para el desarrollo de su competencia comunicativa en idioma inglés hacen énfasis en la necesidad de crear espacios donde se pueda intensificar su uso lo que incrementaría la motivación hacia su aprendizaje.

Según (Quesada, Finalet, Recino, \& Fleite, 2015) considera que algunas regularidades permitieron a los autores concluir que existe un interés manifiesto por parte de los estudiantes y profesionales de la salud en incrementar la práctica de la lengua extranjera, por cuanto es de gran importancia para su formación y desarrollo; se hace necesario incentivar la participación en distintas actividades curriculares y extracurriculares donde se utilice el inglés y que cumplan con las expectativas de los componentes personales del proceso docente educativo para así lograr la motivación necesaria, guiar su atención y desarrollar habilidades comunicativas en este sentido.

Los actos de habla son solamente uno de los muchos factores lingüísticos que pueden afectar a la comunicación. Nos dicen que hay muchas dimensiones que se someten a normas y reglas particulares a un idioma y es necesario que los estudios e investigaciones en el campo de la comunicación y educación English Language Learners in professional nursing careerextranjera donde se proporciona una base común para la elaboración de programas de lenguas, orientaciones curriculares, exámenes, manuales, dramatizaciones, etc., en algunos países describen de forma integradora lo que tienen que aprender a hacer los estudiantes con el fin de utilizar una lengua para comunicarse, así como los conocimientos y destrezas que tienen que desarrollar para poder actuar de manera eficaz.

De acuerdo al criterio de (Pacheco, 2013) considera que el inglés es el lenguaje fundamental de libros, periódicos, aeropuertos y control del tráfico aéreo, negocios internacionales, conferencias académicas, ciencia, tecnología, diplomacia, deportes, música popular y propaganda. Por lo que alrededor de dos tercios de científicos del mundo son los que leen en inglés; y al menos tres cuartos 
Rev SINAPSIS, Vol. 6, Nº 1, Junio 2015

de la información electrónica almacenada está en este idioma, motivo por el cual incentiva al profesional y alumnado en autoeducarce para mejorar su proceso de aprendizaje.

En la realización de este trabajo metodológico, es asumir las constantes transformaciones que se suceden en la educación superior tales como la implementación de las estrategias curriculares y donde se puntualiza que un buen diseño metodológico de las estrategias no es suficiente sino también su organización y funcionamiento correcto y sistemático.

Con relación a la interdisciplinariedad la propuesta se sustenta en este precepto como una herramienta importante en el aprendizaje, para lograr una adecuada relación entre las diferentes asignaturas que conforman el plan de estudio, influye en el consecuente incremento de la efectividad de la enseñanza tanto en términos cuantitativos como cualitativos, con la secuela de una óptima preparación de los estudiantes y del profesor al tener que interactuar con varias disciplinas para dirigir con efectividad el proceso docente-educativo.

\section{Conclusiones}

El inglés con propósitos específicos está diseñado para aprendices que poseen una razón profesional para aprender inglés, así como un contexto común de uso, un conocimiento del contenido de su área; por lo que el docente debe utilizar estrategias mediante las cuales integra las habilidades lingüísticas en el campo vocacional y profesional; así como estrategias de aprendizaje bien desarrolladas.

Según la opinión de los autores consultados, la estrategia es factible de aplicarse para que los profesionales en enfermería y los profesores de las asignaturas logren una adecuada implementación de la estrategia curricular de inglés en cada carrera, y la innegable afirmación de que este idioma constituye una necesidad en los profesionales en la Salud.

\section{Bibliografía}

1. Almudena, A. A., Perez, A. C., \& Albéniz, L. C. (2015). La formacion practica en enfermeria en la escuela universitaria dela comunidad de Madrid. Revista Española de Salud Pública, volumen 76. 


\section{Rev SINAPSIS, Vol. 6, Nº 1, Junio 2015}

2. Camacho, B. R., Barquero, G. A., \& Crespo, M. (2013). El inglés en el grado de enfermeria una asignatura pendiente. Latino-america Enfermagen.

3. Camacho, B. R., Barquero, G. A., Isabel, M. C., \& Merino, N. D. (2013). El Ingles en el grado de Enfermeria: Una asignatura pendiente. Latino America Enfermagen, 3-9.

4. Camacho, B. R., Barquero, G. A., Mariscal, C. M., \& Merino, N. D. (2013). El Inglés en el grado de enfermeria: Una asignatura pendiente. Latino Americana Enfermagen, 3-9.

5. Camacho, B. R., Barquero, G. A., Mariscal, M. I., \& Merino, N. D. (2013). El Ingles en el grado de Enfermeria. Latino Americana, Enfermagen, 3-8.

6. Hernandez, C. I., Cardenas, J., Sanchez, D., \& Torres, P. D. (2012). La enseñanza Posgraduada: Garantia Para aprender el idioma Inglés con propósito específico en Enfermeria. Edumecentro, 39.

7. Jacobson, H. (2011). Lo que crees que has dicho no es lo que creen que has dicho. Panace, 88-88.

8. Montané, G. B., Trujillo, C. O., Cruz, G. H., Carlos, C. P., \& Bosque, C. M. (2012). Estrategia para la superacion profesional en Inglés Medico para profesores en Inglés general. Ciencias Medicas, 4-15.

9. Niño, P. M. (2013). El Inglés y su importancia en la Investigacion Científica. Colombiana ciencia Anim., 7-8.

10. Pacheco, J. A. (2013). ¿Como estimular el desarrollo de la competencia comunicativa en idioma ingles? Edumecentro 2(3), 43-49.

11. Perez, A. C., Alameda, C. A., \& Albéniz, L. C. (2015). La formacion practica en enfermeria en la escuela universitaria de la comunidad de Madrid. Revista Española de Salud Publica, 3-9.

12. Pibaque, P. M., Quevedo, A. N., \& Colunga, S. S. (2015). Analisis histórico de Inglés con fines específico en Ecuador. transformación Issn, 7-12.

13. Pibaque, P. M., Quevedo, A. N., \& Colunga, S. S. (2015). Analisis histórico de Inglés con fines específico en Ecuador. transformación Issn, 11-12.

14. Quezada, L. A., Finalet, M. E., Recino, P. U., \& Fleite, G. Y. (2015). Acciones Metodológica para incentivar el uso del inglés en estudiantes y Profesionales en ciencias Medicas. Edumecentro, 5-9.

15. Quezada, L. A., Finalet, M. E., Recino, P. U., \& Yagima, F. G. (2015). Acciones metodológica para incentivar el uso del Inglés en estudiantes y profesionales de ciencias médicas. Edumecentro, 3-9. 